\title{
Mean-field theory of the spiral phases of a doped antiferromagnet
}

\author{
C. L. Kane, ${ }^{*}$ P. A. Lee, and T. K. Ng ${ }^{\dagger}$ \\ Department of Physics, Massachusetts Institute of Technology, Cambridge, Massachusetts 02139 \\ B. Chakraborty and N. Read \\ Department of Applied Physics, Yale University, New Haven, Connecticut 06520
}

(Received 11 October 1989)

\begin{abstract}
We develop a Schwinger boson-slave fermion mean-field theory for the $t-t^{\prime}-J$ model, where $t^{\prime}$ represents same sublattice hopping. In an expansion for small hole concentration $\delta$, we show that doping favors a spiral distortion of the spins in which the antiferromagnetic order parameter spirals with a wave vector proportional to $\delta$. In addition, we point out the novel possibility of a double spiral state which has isotropic spin correlations, is disordered for arbitrarily small $\delta$, and exhibits a staggered chiral order. The relationship with resonating-valence-bond theory and the implications for neutron scattering experiments are discussed.
\end{abstract}

The interplay between doping and antiferromagnetism is a central issue in theories of the oxide superconductors. Undoped $\mathrm{La}_{2} \mathrm{CuO}_{4}$ is known to be an antiferromagnetic insulator, and may be modeled by $\mathrm{CuO}_{2}$ planes with a spin- $\frac{1}{2}$ local moment on each $\mathrm{Cu}$. $\mathrm{Sr}$ doping introduces holes into the oxygen $p$ orbitals. Following Anderson's suggestion, ${ }^{1}$ there has been considerable interest in the " $t-J$ " model of a doped antiferromagnet

$$
\begin{aligned}
H= & -t \sum_{\langle i j\rangle}\left(1-n_{i-\sigma}\right) c_{i \sigma}^{\dagger} c_{j \sigma}\left(1-n_{j-\sigma}\right) \\
& +J \sum_{\langle i j\rangle}\left(\mathbf{s}_{i} \cdot \mathbf{s}_{j}-\frac{1}{4} n_{i} n_{j}\right),
\end{aligned}
$$

constrained to the subspace with no double occupancy. This follows from the large- $U$ limit of a single-band Hubbard model. Zhang and Rice ${ }^{2}$ have argued that such a model is a good description of the $\mathrm{CuO}_{2}$ planes, since oxygen holes may form a singlet complex with copper moments, which may be considered a hole in a square lattice of copper orbitals.

At half filling this model describes a spin- $\frac{1}{2}$ Heisenberg antiferromagnet on a square lattice which is believed to have long-range Néel order at zero temperature. Away from half filling, the model is characterized by a competition between $t$ and $J$. $J$ favors antiferromagnetic order for the spins, whereas in a Néel state, $t$ will tend to disrupt the spin order, since as it hops, a hole will leave a trail of spins pointing in the wrong direction. ${ }^{3}$ Quantum fluctuations may repair a pair of overturned spins, which allows the holes to hop coherently on the same sublattice. This gives rise to a doubly degenerate band in the reduced Brillouin zone for holes with minima at $\mathbf{k}=( \pm \pi / 2, \pi / 2)$. For $t<J$ the bandwidth is $t^{2} / J$, whereas for $J<t$, incoherent processes renormalize the hole spectrum and there is a coherent band centered at $\approx-t$ with width $\approx J .{ }^{4,5}$ These processes may be treated in an effective way by considering a renormalized model, which contains a samesublattice hopping term, $\sum_{i j} t_{i j}^{\prime} c_{i \sigma}^{\dagger} c_{j \sigma}$. This leads us to study the more general " $t-t$ ' $J$ " model. The parameters in this model may be strongly renormalized from their bare values, and based on our experience with a single hole, ${ }^{4}$ we expect that for $t>J$ the renormalized parameters should be qualitatively given by $t \approx t^{\prime} \approx J$. We emphasize that the physics of $t^{\prime}$ is very different from that of $t$, since a hole may propagate coherently on the same sublattice without disturbing the spins.

Further energy may be gained if holes can hop coherently onto the opposite sublattice which will split the degeneracy between the two sublattices. This is only possible if neighboring spins are not completely antiparallel, so they have nonzero overlap. One way which this may be accomplished is for the spins to cant [Fig. 1(a)]. This is precisely the mechanism for Nagaoka ferromagnetism. ${ }^{6}$ Recently, Shraiman and Siggia ${ }^{3}$ pointed out that it is also possible for the spins to form a spiral [Figs. 1(b)-1(d)]. In a classical theory, they showed that under certain conditions the lowest-energy configuration of the spins is a spiral with a pitch $Q \approx \delta t / J$.

In this paper we are interested in the effects of quantum fluctuations in such states. We develop a mean-field theory based on a slave fermion-Schwinger boson representa-

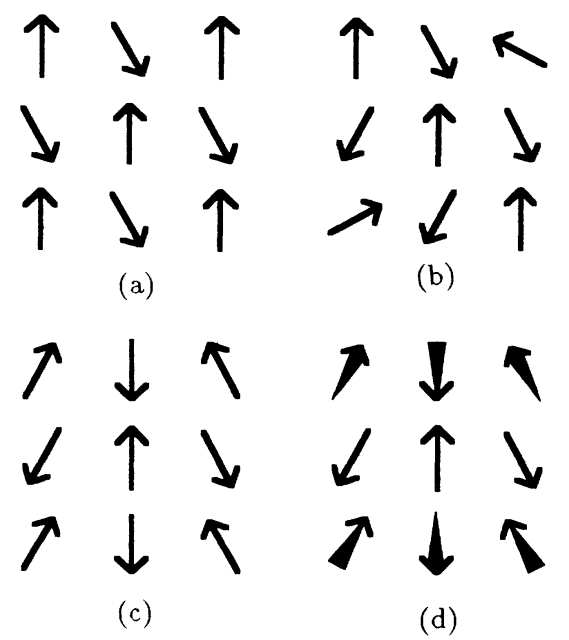

FIG. 1. Schematic pictures of the spin configuration in (a) canting state, (b) $(1,1)$ spiral state, (c) $(1,0)$ spiral state, and (d) double-spiral state. In (d) the small end of the spins are meant to be pointing into the page. 
tion of the $t-t^{\prime}-J$ model, which allows us to treat the constraint forbidding double occupancy in a straightforward approximation:

$$
\begin{aligned}
H= & -t \sum_{\langle i j\rangle} f_{i} f_{j}^{\dagger} b_{i \sigma}^{\dagger} b_{j \sigma}+\sum_{i j} t_{i j}^{\prime} f_{i} f_{j}^{\dagger} b_{i \sigma}^{\dagger} b_{j \sigma} \\
& +\frac{1}{4} J \sum_{\langle i j\rangle} b_{i \alpha}^{\dagger} b_{i \beta} b_{j \gamma}^{\dagger} b_{j \delta}\left(\sigma_{\alpha \beta} \cdot \sigma_{\gamma \delta}-\delta_{\alpha \beta} \delta_{\gamma \delta}\right) \\
& +\sum_{i} \lambda_{i}\left(b_{i \sigma}^{\dagger} b_{i \sigma}+f_{i}^{\dagger} f_{i}-2 S\right)
\end{aligned}
$$

The Schwinger boson operators $b_{i \sigma}^{\dagger}$ keep track of the spins, while the slave fermion operators $f_{i}^{\dagger}$ keep track of the holes. We introduce the Lagrange multiplier $\lambda_{i}$ on each site to enforce the constraint that the total number of fermions and bosons on each site be $2 S$. We consider general spin $S$. For large $S$, quantum fluctuations are suppressed, and our theory reduces to that of Shraiman and Siggia. ${ }^{3}$ This mean-field theory is meaningless in the limit $t>J$, since it ignores the incoherent processes which are known to be important in that limit; ${ }^{4}$ however, we may get qualitative information by considering the renormalized model with $t \approx t^{\prime} \approx J$. Similar mean-field theories of the $t-J$ model have recently been studied by Yoshioka ${ }^{7}$ and by Jayaprakash, Krishnamurthy, and Sarker. ${ }^{8}$

At half filling there are no fermions, and (2) reduces to the Schwinger boson representation of the Heisenberg model. Mean-field theories based on this representation have been very successful at describing the low-temperature properties of the Heisenberg model. ${ }^{9,10}$ The Schwinger boson mean-field approximation to the Heisenberg model amounts to treating $\lambda_{i}$ as a constant independent of position and decoupling the spin interaction in a Hartree-like approximation by introducing the order parameter $D_{\hat{\eta}}=\left\langle b_{i}^{A} b_{i+\hat{\eta} !}^{B}-b_{i}^{A} b_{i+\hat{\eta} \dagger}^{B}\right\rangle$ on each bond. (We consider two sublattices $A$ and $B$ with $i \in A$, so that there are four kinds of bonds, $\hat{\boldsymbol{\eta}}= \pm \hat{\mathbf{x}}, \pm \hat{\mathbf{y}}$.) The resulting Hamiltonian is diagonalized by Bogoliubov transformation. For $S<S_{c}=0.19$ there is a gap in the mean-field boson excitation spectrum, and the spins have a finite correlation length. For $S>S_{c}$ the gap closes, and in two dimensions the Schwinger bosons Bose condense at zero temperature, ${ }^{10}$ which signifies long-range antiferromagnetic order. In what follows, we will consider $T=0^{+}$, in which case there is an exponentially small gap, and the rotational invariance of the theory is not broken. In this case there will be "almost Bose condensation" of the bosons in the vicinity of the gap.

One effect of a finite hole concentration in this meanfield theory is to reduce the effective spin, since the constraint specifies that $b_{i \sigma}^{\dagger} b_{i \sigma}=2 S-\delta$. In the limit $t, t^{\prime} \rightarrow 0$, for $\delta>\delta_{c} \equiv 2\left(S-S_{c}\right)$, Néel order is destroyed by quantum fluctuations. For $S=\frac{1}{2}$, using $S_{c}=0.19^{9}$, we obtain $\delta_{c}=0.61$; however, $\delta_{c}$ may be smaller if in a more accurate treatment of the fluctuations increases the critical spin closer to $\frac{1}{2}$. We thus regard $\delta_{c}$ as a continuous parameter in our mean-field theory.

In addition, the form of the hopping term suggests that we introduce the new order parameters $Q_{\hat{\eta}} \equiv\left\langle b_{i}^{A^{\dagger}} b_{i}^{B}-\hat{\eta} \dagger\right.$ $\left.+b_{i}^{A}{ }^{\dagger} b_{i+\hat{\eta} l}^{B}\right\rangle$ and $F_{\hat{\eta}} \equiv\left\langle f_{i}^{A \dagger} f_{i+\hat{\eta}}^{B}\right\rangle$. We consider uniform $D_{\hat{\eta}}, Q_{\hat{\eta}}$, and $F_{\hat{\eta}} . Q_{\hat{\eta}}$ is the order parameter which describes canting and spiral, since it is nonzero when neigh- boring spins have finite overlap. In order to classify different states in terms of their order parameters $D_{\hat{\eta}}$ and $Q_{\hat{\eta}}$, it is convenient to consider classical (large $S$ ) spins. If we choose a coordinate system in which spin $i$ points up we may parametrize spin $i$ and its neighbors as

$$
\begin{aligned}
& b_{i \sigma}=(2 S)^{1 / 2} e^{i x_{i}}\left(\begin{array}{l}
1 \\
0
\end{array}\right), \\
& b_{i+\hat{\eta} \sigma}=(2 S)^{1 / 2} e^{i x_{i}+\hat{\eta}}\left(\begin{array}{c}
e^{-i \phi_{\hat{\eta}}} \cos \frac{1}{2} \theta_{\hat{\eta}} \\
\sin \frac{1}{2} \theta_{\hat{\eta}}
\end{array}\right),
\end{aligned}
$$

where $\left(\theta_{\hat{\eta}}, \phi_{\hat{\eta}}\right)$ are the spherical coordinates of spin $i+\hat{\eta}$, and $\chi_{i}$ and $\chi_{i+\hat{\eta}}$ are arbitrary phases. Then

$$
Q_{\hat{\eta}}=2 S \exp \left[i\left(\chi_{i+\hat{\eta}}-\chi_{i}-\phi_{\hat{\eta}}\right)\right] \cos \frac{1}{2} \theta_{\hat{\eta}}
$$

and

$$
D_{\hat{\eta}}=2 S \exp \left[i\left(\chi_{i+\hat{\eta}}+\chi_{i}\right)\right] \sin \frac{1}{2} \theta_{\hat{\eta}} .
$$

In a Néel state $\theta_{\hat{\eta}}=\pi$, so that $Q_{\hat{\eta}}=0$. There is a gauge freedom in choosing the phases of $Q_{\hat{\eta}}$ and $D_{\hat{\eta}}$. For example, states with $D_{\hat{\eta}}=D_{-\hat{\eta}}, Q_{\hat{\eta}}= \pm Q_{-\hat{\eta}}$, and $F_{\hat{\eta}}= \pm F_{-\hat{\eta}}$ are related to those with $D_{\hat{\eta}}=-D_{-\hat{\eta}}, Q_{\hat{\eta}}=\mp Q_{-\hat{\eta}}$, and $F_{\hat{\eta}}=\mp F_{-\hat{\eta}}$ via a gauge transformation of the form $b_{i \sigma} \rightarrow b_{i \sigma} e^{i a_{i}^{\hat{\eta}}}, f_{i} \rightarrow f_{i} e^{i a_{i}}$ with $\alpha_{i}=\pi$ for $i=(m \hat{\mathbf{x}}, n \hat{\mathbf{y}})$ such that $m+n=0$ or $1 \bmod 4$, and $\alpha_{i}=0$ otherwise. Such a gauge transformation will also shift the minimum of the fermion band by $(\pi / 2, \pi / 2)$. In the calculations that follow, we work in a gauge in which $D_{\hat{\eta}}$ has $s$ symmetry ${ }^{11}$ $\left(D_{\hat{\eta}}=D\right)$, which corresponds to $\chi_{i}=\chi_{i+\hat{\eta}}=0$. In this gauge, the minimum of the fermion band structure will correspond to that of the $t^{\prime}$ band structure.

The different physical states may be distinguished by the gauge-invariant quantities $P_{\hat{\eta}, \hat{\eta}^{\prime}} \equiv D_{\hat{\eta}}^{*} Q_{\hat{\eta}} D_{\hat{\eta}^{\prime}} Q_{\hat{\eta}^{\prime}}^{*}$. In terms of the classical spins,

$$
P_{\hat{\eta}, \hat{\eta}^{\prime}}=4 S^{4} \sin \theta_{\hat{\eta}} \sin \theta_{\hat{\eta}^{\prime}} \exp \left[i\left(\phi_{\hat{\eta}^{\prime}}-\phi_{\hat{\eta}}\right)\right]
$$

From Fig. 1(a), we see that in the canted state $\phi_{\hat{\eta}}=\phi$, so that $\boldsymbol{P}_{\hat{\eta},-\hat{\eta}}=\boldsymbol{P}_{\hat{\eta}, \hat{\eta}}$. In addition $\boldsymbol{P}_{\hat{\boldsymbol{\lambda}}, \hat{\mathrm{y}}}=\boldsymbol{P}_{\hat{\eta}, \hat{\eta}}$, which is real and positive. In the gauge $D_{\hat{\eta}}=D$, this corresponds to $Q_{\hat{\eta}}$ with $s$ symmetry. The spiral states [Figs. 1(b)-1(d)] are characterized by $\phi_{\hat{\eta}}=\phi_{-\hat{\eta}}+\pi$ or $P_{\hat{\eta},-\hat{\eta}}=-P_{\hat{\eta}, \hat{\eta}}$. The three cases may be distinguished by $\boldsymbol{P}_{\hat{\boldsymbol{t}}, \hat{\boldsymbol{y}}}$. A spiral in the (1,1) direction [Fig. 1(b)] has $\boldsymbol{P}_{\hat{\mathrm{x}}, \hat{\mathrm{y}}}=\boldsymbol{P}_{\hat{\mathrm{y}}, \hat{\eta}}$, and is characterized by $Q_{\hat{\eta}}$ with $p_{x}+p_{y}$ symmetry $\left(\hat{Q}_{\hat{\mathrm{x}}}=Q_{\hat{\mathrm{y}}}=-Q_{-\hat{\mathrm{x}}}\right.$ $=-Q_{-\hat{y}}$. A spiral along the $(1,0)$ direction [Fig. $\left.1(\mathrm{c})\right]$ has $P_{\hat{k}, \hat{y}}=0$, and corresponds to $Q_{\hat{\eta}}$ with $p_{x}$ symmetry $\left(Q_{\hat{\mathrm{x}}}=-Q_{-\hat{\mathrm{x}}}, Q_{ \pm \hat{\mathrm{y}}}=0\right)$. Another interesting possibility is the "double spiral" state in which, locally, the spins spiral in orthogonal planes in the $\hat{\mathbf{x}}$ and $\hat{\mathbf{y}}$ directions [Fig. 1(d)]. Since $\phi_{\hat{\mathrm{z}}}-\phi_{\hat{\mathrm{y}}}=\pi / 2$, this state has $\boldsymbol{P}_{\hat{\mathrm{t}} \hat{\mathrm{y}}}=i \boldsymbol{P}_{\hat{\eta}, \hat{\eta}}$ and is described by $Q_{\hat{\eta}}$ with $p_{x}+i p_{y} \equiv p$ symmetry $Q_{\hat{\mathbf{y}}}=i Q_{\hat{\mathbf{x}}}$.

Before we discuss the properties of these states we briefly outline the structure of the mean-field theory. After a Hartree-Fock decoupling of the Heisenberg and hopping terms the mean-field equations follow from the constraint imposed by $\lambda$ and the self-consistency of $D_{\hat{\eta}}$, $Q_{\hat{\eta}}$, and $F_{\hat{\eta}}$. For spiral states they have the form 


$$
\begin{aligned}
& 2 S+1-\delta=\sum_{k} \frac{\lambda+t_{b \mathbf{k}}^{\prime}+\left|M_{\mathbf{k}}\right|}{2 \omega_{\mathbf{k}}^{+}} \operatorname{coth} \frac{\omega_{\mathbf{k}}^{+}}{2 T}+\frac{\lambda+t_{b \mathbf{k}}^{\prime}-\left|M_{\mathbf{k}}\right|}{2 \omega_{\mathbf{k}}^{-}} \operatorname{coth} \frac{\omega_{\mathbf{k}}^{-}}{2 T}, \\
& D_{\hat{\eta}}=\sum_{k} e^{-i \mathbf{k} \cdot \hat{\eta}}\left(\frac{\frac{1}{2} J D_{\mathbf{k}}}{2 \omega_{\mathbf{k}}^{+}} \operatorname{coth} \frac{\omega_{k}^{+}}{2 T}+\frac{\frac{1}{2} J D_{\mathbf{k}}}{2 \omega_{\mathbf{k}}^{-}} \operatorname{coth} \frac{\omega_{\mathbf{k}}^{-}}{2 T}\right), \\
& Q_{\hat{\boldsymbol{\eta}}}=\sum_{k} e^{-i \mathbf{k} \cdot \hat{\eta}} \frac{M_{\mathbf{k}}}{\left|M_{\mathbf{k}}\right|}\left[\frac{\lambda+t_{b \mathbf{k}}^{\prime}+\left|M_{\mathbf{k}}\right|}{2 \omega_{k}^{+}} \operatorname{coth} \frac{\omega_{\mathbf{k}}^{+}}{2 T}-\frac{\lambda+t_{b \mathbf{k}}^{\prime}-\left|M_{\mathbf{k}}\right|}{2 \omega_{\mathbf{k}}^{-}} \operatorname{coth} \frac{\omega_{\mathbf{k}}^{-}}{2 T}\right), \\
& F_{\hat{\eta}}=\sum_{k} e^{-i \mathbf{k} \cdot \hat{\eta}} \frac{Q_{\mathbf{k}}}{2\left|Q_{\mathbf{k}}\right|}\left[f\left(E_{\mathbf{k}}^{+}\right)-f\left(E_{\mathbf{k}}^{-}\right)\right] .
\end{aligned}
$$

Here we have defined $Q_{\mathbf{k}}=\sum_{\hat{\eta}} e^{i \mathbf{k} \cdot \hat{\eta}} Q_{\hat{\eta}}$ (and likewise for $D_{\mathbf{k}}$ and $\left.F_{\mathbf{k}}\right)$ and $M_{\mathbf{k}}=\frac{1}{4} J Q_{k}+t F_{\mathbf{k}}$. Thus,

$$
t_{b \mathbf{k}}^{\prime}=\sum_{\mathbf{k}} t_{\mathbf{k}-\mathbf{k}^{\prime}}^{\prime}\left[f\left(E_{\mathbf{k}}^{+}\right)+f\left(E_{\mathbf{k}}^{-}\right)\right] \approx \delta t_{\mathbf{k}-\mathbf{k}_{0}}^{\prime}
$$

where $t_{\mathbf{k}}^{\prime}$ is the band structure corresponding to $t_{i j}^{\prime}$. For example, if there is only second- and third-neighbor hopping $t_{1}^{\prime}$ and $t_{2}^{\prime}$, it will be

$$
t_{\mathrm{k}}^{\prime}=4 t_{2}^{\prime} \cos k_{x} \cos k_{y}+2 t_{3}^{\prime}\left(\cos 2 k_{x}+\cos 2 k_{y}\right)
$$

The minimum of this band structure is at $\mathbf{k}_{0}=( \pm \pi / 2$, $\pi / 2)$ when $t_{3}^{\prime}>\frac{1}{2}\left|t_{2}^{\prime}\right|$. The mean-field dispersion for the two branches of bosons is

$$
\omega_{\mathbf{k}}^{ \pm}=\left[\left(\lambda+t_{b \mathbf{k}}^{\prime} \pm\left|M_{\mathbf{k}}\right|\right)^{2}-\left|\frac{1}{2} J D_{\mathbf{k}}\right|^{2}\right]^{1 / 2} .
$$

In the canted state the mean-field equations have a dif- ferent form. The boson dispersion is

$$
\omega_{\mathbf{k}}^{ \pm}=\left[\left(\lambda+t_{b \mathbf{k}}^{\prime}\right)^{2}-\left|\frac{1}{2} J D_{\mathbf{k}}\right|^{2}\right]^{1 / 2} \pm\left|M_{\mathbf{k}}\right| .
$$

The fermion band structure in both cases is given by $E_{\mathbf{k}}^{ \pm}=t_{f \mathbf{k}}^{\prime} \pm t\left|Q_{\mathbf{k}}\right|$, where $t_{f \mathbf{k}}^{\prime}=\sum_{\mathbf{k}^{\prime}} t_{\mathbf{k}-\mathbf{k}^{\prime}}^{\prime}\left\langle b_{\mathbf{k}^{\prime} \sigma}^{A^{\dagger}} b_{\mathbf{k}^{\prime} \sigma}^{A}\right\rangle$. The minimum of the fermion band structure will be at $( \pm \pi / 2, \pi / 2)$ as long as $t_{3}^{\prime}>\frac{1}{2}\left|t_{2}^{\prime}\right|\left(0.28+\delta_{c}\right) /(0.22$ $\left.+\delta_{c}\right)$. Coupling to $Q_{\hat{\eta}}$ induces a splitting in the fermion band structure, which causes a polarization $F_{\hat{\eta}}$ with the same symmetry as $Q_{\hat{\eta}}$. Canting will cause splitting predominantly at $\mathbf{k}=(0,0)$ and $(0, \pi)$, whereas spiral causes splitting at $\mathbf{k}=( \pm \pi / 2, \pi / 2)$.

For the case $\delta=0$, our equations correspond exactly to those obtained earlier. ${ }^{9,10}$ We have performed an expansion for small $\delta$ at $T=0^{+}$and analytically obtained the leading nontrivial corrections. Care must be taken in evaluating the sums in the regions where $\omega_{\mathbf{k}} \approx 0$. We consider four kinds of states: canting, $(1,1)$ spiral, $(1,0)$ spiral, and $(1, i)$ double spiral. The energy differences between these states and the $Q_{\hat{\eta}}=0$ state are

$$
\begin{aligned}
& \Delta E_{\text {cant }}=\delta^{2}\left(-\frac{t^{2}}{J} \frac{8}{1+2\left(I_{1}+\delta_{c}\right) / \delta_{c}}+\frac{3}{2} \rho^{-1}\right)=\delta^{2}\left(-1.67 \frac{t^{2}}{J}+\frac{3}{2} \rho^{-1}\right), \\
& \Delta E_{(1,1)}=\delta^{2}\left(-\frac{t^{2}}{J} \frac{8}{1+2\left(I_{1}+\delta_{c}\right) /\left(I_{2}+\delta_{c}\right)}+\frac{3}{2} \rho^{-1}\right)=\delta^{2}\left(-2.20 \frac{t^{2}}{J}+\frac{3}{2} \rho^{-1}\right), \\
& \Delta E_{(1,0)}=\delta^{2}\left(-\frac{t^{2}}{J} \frac{4}{1+2\left(I_{1}+\delta_{c}\right) /\left(I_{2}+\delta_{c}\right)}+\frac{1}{2} \rho^{-1}\right)=\delta^{2}\left(-1.10 \frac{t^{2}}{J}+\frac{1}{2} \rho^{-1}\right), \\
& \Delta E_{(1, i)}=\delta^{2}\left(-\frac{t^{2}}{J} \frac{4}{1+2\left(I_{1}+\delta_{c}\right) /\left(I_{2}+\delta_{c} / 2\right)}+\frac{1}{2} \rho^{-1}\right)=\delta^{2}\left(-0.79 \frac{t^{2}}{J}+\frac{1}{2} \rho^{-1}\right),
\end{aligned}
$$

where $\rho$ is the density of states at the bottom of the fermion band $t_{f k}^{\prime}$. $I_{1}=0.54$ and $I_{2}=0.26$ are numerical integrals. The expressions on the right-hand side refer to the case $S=\frac{1}{2}$. In a pure $t-J$ model, with $t^{\prime}=0\left(\rho^{-1}=0\right)$ we find that the $(1,1)$ spiral state has the lowest energy. Classically, this spiral state and the canting state have the same energy, as can be seen from the large- $S$ limit. However, quantum fluctuations favor the spiral over the canting. For $t^{\prime} \neq 0$, there are different optimal solutions depending on $\rho$. Assuming the minimum is at $( \pm \pi / 2, \pi / 2)$, for $\rho t^{2} / J>0.91$ the $(1,1)$ spiral is favored, whereas for $0.91>\rho t^{2} / J>0.45$ the $(1,0)$ spiral has lower energy. For $\rho t^{2} / J<0.45$, the spiral states are unstable relative to the Néel state. In the parameter regime where the $(1,0)$ spiral state is favorable, the double-spiral state is competi- tive. The difference in energy between the two decreases for smaller $\delta_{c}$. (5) is valid when $\delta_{c}>0$ and $\delta \ll \delta_{c}$. A separate calculation shows that if $\delta_{c}<0$ (i.e., $S<S_{c}$ ) and $\delta \ll\left|\delta_{c}\right|$ then the $(1,0)$ state and the $(1, i)$ state are degenerate.

At long distances, the spin correlations are dominated by the low-energy modes where $\omega_{k} \approx 0$. In the $(1,0)$ and $(1,1)$ states, $\omega_{\mathbf{k}} \approx \sqrt{8} J D\left|\mathbf{k}-\mathbf{k}_{0}\right|$ for $\mathbf{k} \approx \mathbf{k}_{0}= \pm 1.26 \delta t /$ $J(1,0)$ or $\pm 1.26 D t / J(1,1)$. At $T=0$ there is Bose condensation at $\mathbf{k}_{0}$ with a density $n_{0} \equiv \delta_{c}-\delta$, which means that there is incommensurate spiral with wavelength $\frac{1}{2} \mathbf{k}_{0}^{-1}$ which has long-range order. For spins on the same sublattice at long distances $\left\langle\mathbf{s}_{i}^{A} \cdot \mathbf{s}_{j}^{A}\right\rangle=\frac{3}{8} n_{0}^{2} \cos \left(2 \mathbf{k}_{0} \cdot \mathbf{r}_{i j}\right)$. In a neutron scattering experiment we would expect to see a Bragg peak separated by $\mathbf{k}_{0}$ from the antiferromagnetic 
wave vector.

The double-spiral state has very interesting properties. If we examine the dispersion of the low-lying excitations, we find that $\omega_{\mathbf{k}}=\sqrt{8} J D\left[\left(|\mathbf{k}|-k_{0}\right)^{2}+\epsilon^{2}\right]^{1 / 2}$ for $|\mathbf{k}|$ $\approx k_{0}=1.40 \delta t / J$. That is, there is a ring of low-lying excitations rather than points. This means that the spin correlations should be isotropic, and that neutron scattering experiments should reveal a Bragg ring around the antiferromagnetic wave vector rather than Bragg peaks. Furthermore, for arbitrarily small $\delta$, there is a finite gap at $T=0, \epsilon \approx \delta J e^{-\delta_{c} / \delta}$. There is thus no Bose condensation, and the spins are disordered with a correlation length $\xi \approx \delta^{-1} e^{\delta_{c} / \delta}$. This happens because the ring of low-lying excitations effectively lowers the dimensionality and melts the long-range order. For $\delta^{-1}<r<\xi$, the staggered spin correlations behave like

$$
\langle\mathbf{s}(0) \cdot \mathbf{s}(r)\rangle \approx \frac{3}{8} n_{0}^{2}\left[J_{0}\left(k_{0} r\right)^{2}-J_{1}\left(k_{0} r\right)^{2}\right] .
$$

Though the double-spiral state has no spin order, it exhibits a hidden, Ising-like chiral order parameter. This may be seen from the fact that the gauge-invariant quantity $\boldsymbol{P}_{\hat{\mathrm{x}}, \hat{\mathrm{y}}}$ is imaginary, and the imaginary part of $\boldsymbol{P}_{\hat{\mathrm{x}}, \hat{\mathrm{y}}}$ may be shown to be related to the chiral order parameter, ${ }^{12}$
$\operatorname{Im} P_{\hat{\mathrm{x}}, \hat{\mathrm{y}}}=4 S\left\langle\mathrm{~s}_{i}^{A} \cdot\left(\mathrm{s}_{i+\hat{\mathrm{x}}}^{B} \times \mathrm{s}_{i+\hat{\mathrm{y}}}^{B}\right)\right\rangle$. Actually this state is characterized by staggered chirality, since translation by one lattice site reverses the sign of $\operatorname{Im} P_{\hat{\mathbf{x}}, \hat{\mathbf{y}}}{ }^{13}$ This is in contrast to the uniform chirality which has been proposed in connection with flux phases and anyon superconductivity. ${ }^{12}$

Though the double spiral has higher mean-field energy than the other spiral states, we believe that it is still a possible candidate. The energy calculated here may not be reliable, since our mean-field theory violates the constraint forbidding double occupancy. Furthermore, fluctuations in the order parameter $Q_{\hat{\eta}}$, which should be related to the "torsion mode" discussed by Shraiman and Siggia, ${ }^{3}$ should behave very differently in the single- and double-spiral states due to the existence of the ring of low-lying excitations.

An alternative approach is to consider variational wave functions which correspond to spiral states. This leads us to an interesting connection with resonating-valence-bond (RVB) theory. ${ }^{1,14}$ Our Hartree-Fock decoupling may be regarded as a variational wave function for an approximate Hamiltonian in which the constraint of no double occupancy is treated on the average. For spiral states, this wave function for the spins is of the form

$$
|\psi\rangle \propto \exp \sum_{k}\left[\frac{1}{2}\left(g_{\mathbf{k}}^{+}+g_{\mathbf{k}}^{-}\right)\left(b_{\mathbf{k} \uparrow}^{A \dagger} b_{-\mathbf{k} \downarrow}^{B^{\dagger}}-b_{\mathbf{k} \dagger}^{A \dagger} b_{-\mathbf{k} \downarrow}^{B \dagger}\right)+\frac{1}{2}\left(g_{\mathbf{k}}^{+}-g_{\mathbf{k}}^{-}\right)\left(s_{\mathbf{k}} b_{\mathbf{k} \uparrow}^{A \dagger} b_{-\mathbf{k} \downarrow}^{A \dagger}-s_{\mathbf{k}}^{*} b_{\mathbf{k} \uparrow}^{B_{\dagger}^{\dagger}} b_{-\mathbf{k} \downarrow}^{B^{\dagger}}\right)\right]|C\rangle,
$$

where $g_{k}^{ \pm}=\left(\omega_{k}^{ \pm}+t_{b k}^{\prime}+\lambda \pm\left|M_{k}\right|\right) / D_{k}, s_{k}=M_{k} /\left|M_{k}\right|$, and $|C\rangle$ is a rotationally averaged classical state which corresponds to the Bose condensed part of the wave function. At half filling $g_{k}^{+}=g_{k}^{-}$and the wave function is composed of singlets between sites on opposite sublattices with a positive weight. If projected to the constrained subspace, this state becomes ${ }^{15}$ a finite-range RVB wave function of the form studied by Liang, Doucot, and Anderson, ${ }^{14}$ in which singlets are placed between sites on different sublattices with phases in correspondence with the Marshall sign rule. The wave function (6) shows that in a spiral state there is also a contribution from singlets between sites on the same sublattice. Furthermore, the distinction between various spiral states is built into the phases of the same sublattice singlets. In particular, in a double-spiral state, since the symmetry of $M_{k}$ is complex $p$ wave, the phase will be $e^{i \theta}$, where $\theta$ is the angle which the bond makes with the $\hat{\mathbf{x}}$ axis. The analogous wave function for the canted state does not contain samesublattice singlets.

"Present address: IBM, Thomas J. Watson Research Center, Yorktown Heights, NY 10598.

'Present address: AT \& T Bell Laboratories, Murray Hill, NJ 07974.

'P. W. Anderson, Science 235, 1196 (1987).

${ }^{2}$ F. C. Zhang and T. M. Rice, Phys. Rev. B 37, 3759 (1988).

${ }^{3}$ B. Shraiman and E. Siggia, Phys. Rev. Lett. 62, 1564 (1989).

${ }^{4}$ C. L. Kane, P. A. Lee, and N. Read, Phys. Rev. B 39, 6880 (1989).

${ }^{5}$ S. Schmitt-Rink, C. Varma, and A. Ruckenstein, Phys. Rev. Lett. 60, 2793 (1988).

${ }^{6}$ Y. Nagaoka, Phys. Rev. 147, 392 (1966).

${ }^{7}$ D. Yoshioka, J. Phys. Soc. Jpn. 58, 1516 (1989).

${ }^{8}$ C. Jayaprakash, H. R. Krishnamurthy, and S. Sarker, Phys. Rev. B 40, 2610 (1989).
Neutron scattering studies ${ }^{16}$ have indicated that there is incommensurate spin order in $\mathrm{La}_{2-x} \mathrm{Sr}_{x} \mathrm{CuO}_{4}$, though at present it is not known whether or not there is a Bragg ring. In addition, the incommensurability seems to appear only in superconducting samples, which lends support to the idea that it is related to the hole mobility.

In summary, we have demonstrated in a simple meanfield theory how doping can induce spiral order in a quantum antiferromagnet. Quantum fluctuations will disorder the spins upon sufficiently large doping, and tend to favor spiral order over canting. In addition, we have pointed out the novel possibility of a double-spiral state, which has isotropic spin correlations, is disordered for arbitrarily small doping and exhibits staggered chirality.

We are grateful to N. Nagaosa and B. Shraiman for stimulating discussions. This work was funded by the $\mathrm{Na}$ tional Science Foundation through the Materials $\mathbf{R e}-$ search Laboratory, Grant No. DMR 87 19217. C.L.K. acknowledges partial financial aid from IBM.

${ }^{9}$ D. P. Arovas and A. Auerbach, Phys. Rev. B 38, 316 (1988); A. Auerbach and D. P. Arovas, Phys. Rev. Lett. 61, 617 (1988).

${ }^{10}$ D. Yoshioka, J. Phys. Soc. Jpn. 58, 32 (1989).

"We do not consider states with $s+i d$ symmetry, since in Refs. 9 and 10 they have been shown to have higher energy at half filling.

${ }^{12}$ X. G. Wen, F. Wilczek, and A. Zee, Phys. Rev. B 39, 1143 (1989).

${ }^{13}$ The existence of staggered chiral order has been independently noted by B. Shraiman and E. Siggia (unpublished).

${ }^{14}$ S. Liang, B. Doucot, and P. W. Anderson, Phys. Rev. Lett. 61, 365 (1988).

${ }^{15}$ N. Read and S. Sachdev, Phys. Rev. Lett. 62, 1694 (1989).

${ }^{16}$ T. R. Thurston et al., Phys. Rev. B 40, 4585 (1989). 